\title{
INVESTIGACIÓN/RESEARCH
}

\section{ASPECTOS CONCEPTUALES SOBRE LA INTELIGENCIA AFECTIVA Y SU FOMENTO A TRAVÉS DE LA LECTURA EN LA ESCUELA}

Valentín Martínez-Otero Pérez ${ }^{\mathbf{1}}$ : Universidad Complutense de Madrid. valenmop@edu.ucm.es

Virginia García-Lago Ibáñez: Colegio Santa María, Collado Villalba (Madrid) vgarcialago@hotmail.com

\section{Resumen}

En este artículo se profundiza en el concepto de "inteligencia afectiva", en sus implicaciones educativas y en su cultivo a través de la lectura. Aunque cada vez se reconoce más la trascendencia de la educación de la afectividad, siguen siendo insuficientes los esfuerzos por desarrollar programas formativos sistemáticos y rigurosos. En este trabajo se ofrecen recomendaciones prácticas dirigidas a educadores y se destaca el valor de la lectura como recurso psicopedagógico para desarrollar la inteligencia afectiva.

Palabras clave: Inteligencia afectiva - Lectura - Educación - Competencia emocional.

\footnotetext{
${ }^{1}$ Autor correspondiente:

Valentín Martínez-Otero Pérez: Profesor del Departamento de Teoría e Historia de la Educación de la Facultad de Educación de la Universidad Complutense de Madrid. España.

Correo: valenmop@edu.ucm.es
} 


\title{
CONCEPTUAL ASPECTS OF EMOTIONAL INTELLIGENCE AND ITS DEVELOPMENT THROUGH SCHOOL READING
}

\begin{abstract}
The affective trend continues being the forgotten subject in the curriculum. The new milenium school cannot give up the challenge of educating in harmony, from the respect to individual differences, all the aspects of one's personality. There are not any widely accepted propositions yet that can lead the pedagogical praxis wich is launched to cultívate such dimension, and the obstacles wich fiad iniciatives of this kind aren't just few. In this article the concept of affective intelligence is analized as to a mixture of racional and emotional processes that has to receive educative attention. Here are some practical recommendations targeted at educators and emphasizes the value of reading as a resource for the development of affective intelligence.
\end{abstract}

Key words: Affective intelligence, affective development, integral education, reading, school.

\section{INTRODUCCIÓN}

Aun cuando hay un mare magnum de definiciones sobre el concepto de inteligencia, cabe agregar la que corresponde a la inteligencia afectiva, definida con brevedad como "la capacidad para conocer, expresar y canalizar la cognición y la afectividad, sobre todo los sentimientos, las emociones, las pasiones y las motivaciones" (Martínez-Otero, 2007).

Veamos con mayor detenimiento los términos y el alcance de esta definición ${ }^{1}$ :

- Conocimiento de la cognición.- Es la capacidad para identificar los procesos de pensamiento. Equivale a hablar de metacognición, toda vez que hay una autovaloración y autorregulación del pensamiento. Es una "lectura interior" que favorece el razonamiento consistente, la flexibilidad cognitiva, el sentido social, la abstracción, la búsqueda de la verdad, la adecuada toma de decisiones, la resolución de problemas, etc.

- Expresión del pensamiento.- El lenguaje permite expresar el pensamiento, pero lo cierto es que no siempre se sabe manifestar lo que se piensa. Cada vez hay más quejas sobre la escasa o perturbada comunicación verbal, incluso en el ámbito de las relaciones de pareja. Ni que decir tiene que muchas dificultades de expresión lingüística están íntimamente relacionadas con la ausencia o endeblez de parlamento interior o soliloquio. En cualquier caso, a través del lenguaje saludable (ad intra y ad extra) estructuramos la

\footnotetext{
${ }^{1}$ De acuerdo a la descripción de Rojas $(1988,17)$, identificamos las cuatro experiencias afectivas fundamentales citadas.
} 
realidad, transmitimos información, entramos en contacto con los otros, influimos en el comportamiento y dilatamos nuestro mundo.

- Gobierno de la cognición. - Es la capacidad para regular constructivamente el pensamiento, esto es, para ponerlo al servicio del entendimiento, la concordia, la verdad y el bien. Esta canalización del pensamiento se ha de advertir en las acciones del sujeto, en sus relaciones interpersonales, ajuste y bienestar psicosocial.

- Conocimiento de la afectividad.- Es la capacidad de identificar los fenómenos afectivos propios y aun ajenos, la aptitud para acceder a la vida emocional y conocer su complejidad. Es la habilidad para discriminar e interpretar correctamente los estados de ánimo. La persona con conocimiento de la afectividad advierte fácilmente la naturaleza de los sentimientos, emociones, pasiones y motivaciones, los relaciona y juzga con acierto.

- Expresión de la afectividad.- Es la capacidad ${ }^{2}$ de manifestar la propia afectividad, es decir, la habilidad para mostrar adecuadamente la experiencia emocional. A semejanza del artista, la persona que sabe expresar su afectividad da a entender con viveza y exactitud sus estados de ánimo. La expresión de la afectividad está condicionada socioculturalmente y, por lo mismo, se requiere conocimiento de los códigos concretos para que los mensajes emocionales se manifiesten e interpreten bien. La expresión emocional está al servicio de la comunicación.

- Control de la afectividad.- Es la capacidad de gobernar las experiencias afectivas, esto es, de adecuarlas a las situaciones y ponerlas al servicio de los proyectos vitales. La persona con control emocional se autodomina, afronta los altibajos de la vida y mantiene el equilibrio afectivo, o sea, la templanza.

Los aspectos descritos proporcionan una idea básica sobre la inteligencia afectiva. Aunque hay personas que poseen distinta competencia en cada uno, la inteligencia afectiva depende de su interrelación. Las implicaciones de la inteligencia afectiva cubren un amplio espectro que se inicia en la propia persona, con toda la riqueza y complejidad del paisaje cognitivo-emocional, y se extiende a las distintas situaciones interhumanas y experiencias vitales; de hecho, la inteligencia afectiva repercute en todos los ámbitos de la vida: familiar, académico, laboral, social, etc. Por eso es tan importante desarrollarla.

\footnotetext{
${ }^{2}$ La dificultad para expresar la afectividad puede conducir a la alexitimia (incapacidad para identificar y verbalizar las emociones). Aunque su aparición depende de distintos factores, parece ser más frecuente en personas inmersas en ambientes que reprimen las manifestaciones afectivas. La alexitimia parece correlacionar con anhedonia social o dificultad para disfrutar de las actividades sociales. También se ha extendido la idea de que nuestra "sociedad tecnificada" ha elevado la incidencia del problema.
} 
Conviene hacer notar que no se trata de un nuevo tipo de inteligencia que se sume a las largas colecciones que tenemos, sino de una necesidad. La estructura intelectual está inevitablemente unida a la afectividad y aun a la moralidad. A este respecto, Piaget (1973) sostiene que, desde el período preverbal, hay un estrecho paralelismo entre el desarrollo de la afectividad y el de las funciones intelectuales, puesto que son aspectos indisociables de cada acción. Es interesante también el estudio del egregio psicólogo suizo sobre las relaciones entre inteligencia y afectividad (2001).

Como dicen Hersh, Reimer y Paolitto (1988), los estadios de desarrollo de Piaget adquieren valor no sólo por describir la estructura del razonamiento científico, sino porque se extienden también al razonamiento en los campos moral y social. Textualmente: "El juicio moral es el área en que Piaget ha tratado más explícitamente la relación entre el conocimiento y el afecto. Porque, ¿qué es el juicio moral sino una estructura cognitiva de cómo sentimos que debemos tratar a otros y de cómo los demás nos deben tratar?"

Kohlberg (1992), por su parte, se expresa en términos parecidos a Piaget y aplicó al estudio del juicio moral el concepto de desarrollo en estadios elaborado por el autor ginebrino.

Lo que parece claro es que hay que cultivar la inteligencia afectiva y, cómo no, la afectividad inteligente, so pena de embrutecernos, por más que se posean algunas habilidades cognitivas relativamente autónomas.

Con frecuencia nos encontramos con algunas personas supuestamente muy inteligentes que son incapaces de ponerse en el lugar de los demás, que no reconocen los estados de ánimo propios ni ajenos y que no saben expresar lo que sienten. Por el contrario, otras personas, a pesar de obtener bajas puntuaciones en las tradicionales pruebas de cociente intelectual, se conducen con equilibrio y manifiestan una inclinación afectiva hacia los otros, habitualmente en forma de simpatía, es decir, propenden a conmoverse espontánea y sinceramente con los sentimientos de los demás.

Estos casos y otros de mayor gravedad están muy extendidos y nos llevan a insistir en la necesidad de revisar el concepto de inteligencia y de diseñar nuevos instrumentos de medida de esta facultad que tengan en cuenta el componente afectivo. Asimismo, dado que la inteligencia afectiva hay que cultivarla, se hace imprescindible modificar la educación actual -demasiado centrada en los contenidos y muy poco en la vertiente emocional-, en aras de la persona en su totalidad.

\section{DESARROLLO TEÓRICO}

Dado que hemos definido sucintamente qué entendemos por "inteligencia afectiva", bueno es que exploremos su naturaleza. Vaya por delante que nos hallamos ante una nueva forma de aproximarnos a esta compleja realidad, por más que el concepto de "inteligencia emocional" haya experimentado un impulso extraordinario, en gran 
medida gracias a los trabajos de Goleman (1997; 2000). La difusión y popularidad alcanzada por estos libros y otros en su estela ha sido realmente espectacular y beneficiosa, pues el impacto de las publicaciones ha llevado a la comunidad científica a tomarse muy en serio una "nueva" línea de investigación que abre las puertas al necesario replanteamiento del concepto de inteligencia. Es cierto que antes de que la inteligencia se diese este "baño de multitudes" ya se trabajaba en la dirección señalada, pero el éxito de las obras mencionadas ha dado un espaldarazo fundamental a algunas iniciativas, acaso tímidas, en el ámbito de la psicología. Ojalá que el resultado feliz no desvíe a la ciencia del comportamiento de sus objetivos perentorios, pues aún es largo el camino que ha de recorrerse hasta llegar a conocer exhaustivamente la "inteligencia afectiva", cómo se puede evaluar y, lo que quizá sea más importante, con qué métodos pedagógicos se desarrolla.

Para dar mayor consistencia y alcance a la propuesta que nos ocupa tal vez sea oportuno elaborar un mapa de la inteligencia afectiva. En su inicio esta descripción orográfica da cuenta de la imbricación de procesos cognitivos y afectivos, toda vez que en nuestra experiencia del mundo es inseparable lo que pensamos de lo que sentimos. Zubiri (1991), v. gr., al referirse a la inteligencia sentiente dice que el sentir humano y la intelección no son dos actos numéricamente distintos, sino que constituyen dos momentos del mismo acto de aprehensión sentiente de lo real.

La captación de la realidad, fruto de la coordinación y equilibrio entre operaciones racionales y emocionales, permite identificar una estructura cognitivo-afectiva. Castilla del Pino (2000), habla de módulo cognitivo-emocional estándar, ya que, como él mismo indica, la experiencia de nuestra relación con un objeto rememorado incluye un componente cognitivo y un componente emocional. Vivir una situación, por ejemplo, implica conocerla y sentirla.

El hecho de reconocer una estructura cognitivo-afectiva, al tiempo que conduce a admitir la combinación de circuitos de doble naturaleza también presupone la aceptación de procesos diferenciados. Asimismo, interesa destacar que el cambio en una parte del entramado modifica la totalidad y a la inversa. Siendo así, la psicopedagogía debe encontrar, tras identificar los elementos del complejo módulo cognitivo-emocional, los cauces apropiados para enriquecerlo, lo que es tanto como promover simultáneamente mudanzas armónicas y positivas en los procesos cognitivos y en los procesos emocionales A tal respecto, la reflexión y la revisión de literatura científica nos llevan a describir tres tipos de competencia -cognitiva, afectiva y conductual- que constituyen la inteligencia afectiva y que, de acuerdo a los fines de nuestro trabajo, poseen valor operativo para su mejora:

- Competencia cognitiva.- Es la capacidad para utilizar el pensamiento de forma eficaz y constructiva. Incluye los procesos mentales de comprensión, razonamiento, abstracción, resolución de problemas, aprendizaje de la experiencia y adaptación al entorno. Especialmente relevante es la metacognición o capacidad para pensar en la propia cognición y controlarla. En el marco de la inteligencia afectiva, la competencia cognitiva se caracteriza por 
una orientación prosocial, pues el conocimiento y la habilidad que se poseen tienden a reforzar los lazos interpersonales, es decir, la convivencia.

Con carácter práctico incluimos en este nivel el razonamiento, en cuanto capacidad de discurrir, facultad de analizar y sintetizar, aptitud para organizar la realidad y resolver problemas, etc. Esta habilidad permite pensar con lógica, juzgar críticamente, interesarse por cuestiones abstractas y problemas del entorno. En definitiva, esta dimensión es un indicador del potencial cognitivo del sujeto.

- Competencia afectiva.- Es la capacidad para reconocer, expresar y canalizar la vida emocional. Adquiere especial importancia el equilibrio personal, la autoestima y la empatía. También es importante la metaafectividad o capacidad del sujeto para conocer y gobernar los sentimientos que provocan los fenómenos afectivos. Este componente se refiere sobre todo a las habilidades que tiene el sujeto para comprender la afectividad $y$, en consecuencia, enriquecer la propia vida.

Desde un punto de vista operativo se enmarcan en este módulo la autoestima, la empatía y la lexitimia:

. La autoestima es el valor que la persona se atribuye a sí misma. Tanta es la trascendencia de la aceptación y el aprecio del self que son requisitos para mantener la homeostasis. La consideración positiva de la propia identidad impulsa a la autorrealización.

- La empatía es la capacidad que permite entender a los demás, generalmente en lo que se refiere a sus emociones. En la empatía es nítida la trabazón entre cognición y afectividad, pues la habilidad para reconocer los estados anímicos ajenos requiere sensibilidad, comprensión, destreza perceptiva y madurez. La empatía es el punto de partida de las relaciones sociales positivas y aun del altruismo.

- La lexitimia es la capacidad para reconocer y expresar la propia afectividad. La persona que posee esta habilidad conoce y manifiesta sus experiencias emocionales adecuadamente en las distintas situaciones, lo que predispone a sintonizar con los demás.

- Competencia conductual.- Son las acciones que realiza el sujeto, a partir de su pensamiento y de su afectividad. La planificación es básica para que no nos hallemos, más allá de lo imprescindible, en meros automatismos. La estructura cognitivo-emocional equilibrada y rica libera de la "robotización" y facilita la aparición de conductas positivas a nivel personal, profesional, social, etc.

Con finalidad aplicada incluimos en esta categoría las habilidades sociales, entendidas como conductas, tanto verbales como no verbales, que permiten a las personas iniciar y mantener relaciones saludables con los demás. Para que se pueda hablar de habilidades sociales es preciso que estas destrezas sean beneficiosas y satisfagan. Se enmarcan dentro de un comportamiento 
aceptado y valorado socialmente que nada tiene que ver con la manipulación engañosa y malsana.

Aunque no hay acuerdo unánime en señalar los componentes de la inteligencia afectiva, entre otras razones porque es poco el tiempo que la comunidad científica lleva interesándose por esta cuestión, con frecuencia los autores que abordan el tema distinguen los tres módulos descritos que, dicho sea de paso, operan entrelazadamente. En función del concepto de inteligencia afectiva que aquí se analiza y propugna los aspectos estrictamente racionales no son "neutros", sino que están cargados de afectividad y empujan a actuar en una determinada dirección: el crecimiento personal y social. Esta ejemplificación, al tiempo que muestra la necesidad de trabajar las tres vertientes, permite comprobar que el tratamiento educativo que reciba la estructura cognitivo-emocional no puede quedar al margen de los valores. Topamos, pues, con la ética, por cuanto el hombre es, por su propia naturaleza, un ser moral. Frente al subjetivismo o relativismo que, en ocasiones, defienden algunos métodos pseudoformativos, defendemos una educación de la inteligencia afectiva impulsora de reflexión, responsabilidad, libertad, creatividad, solidaridad y convivencia. Bueno es que se explicite este marco axiológico general, para que no haya dudas sobre el rumbo que ha de tomar cualquier programa psicopedagógico que se precie.

La naturaleza de la inteligencia afectiva es rica en extremo y explica, por ejemplo, aspectos como la organización axiológica de la realidad, el fortalecimiento de la propia identidad, la búsqueda de la verdad y el bien, etc., procesos que apenas serían comprensibles si se invoca únicamente una vertiente de la estructura que nos ocupa. Sea como fuere, el engarce entre el sistema cognitivo-afectivo y la moral permanece todavía oculto en gran medida y no sería extraño que, en plazo breve, se impulse la investigación encaminada a su esclarecimiento, algo que acaso permitiría hablar, pese a la radical oposición de algunos, de "inteligencia moral".

\section{ALCANCE PRÁCTICO}

Se acepta que las personas con elevada inteligencia afectiva tienen más posibilidades de adaptarse a las situaciones y de obtener éxito en los proyectos. En el ámbito laboral, por ejemplo, se está tomando conciencia de la importancia que la competencia cognitivo-emocional tiene en la satisfacción y el rendimiento en el trabajo, hasta el punto de que han surgido algunas iniciativas de formación empresarial encaminadas a promoverla en las organizaciones. Huelga decir que en la institución escolar también se está reconociendo la trascendencia de la inteligencia afectiva, aun cuando siguen siendo insuficientes los esfuerzos por desarrollar programas sistemáticos y rigurosos.

Según refiere Goleman 1997, los resultados obtenidos tras la aplicación de algunos programas encaminados a favorecer el aprendizaje social y emocional en escuelas norteamericanas revelan que los escolares que han participado en el proceso se tornan más responsables, asertivos, colaboradores, comprensivos, eficaces, tolerantes, pacíficos, respetuosos, sensibles y prosociales que los alumnos que no han seguido 
ningún entrenamiento especial. Las mismas investigaciones indican que estos proyectos de desarrollo personal mejoran el clima social del aula, reducen las conductas violentas y la delincuencia, al tiempo que aumentan los vínculos positivos de los niños y adolescentes con las respectivas familias y escuelas

La investigación actual confirma que los programas educativos cuya finalidad es el desarrollo de las capacidades socioemocionales mejoran significativamente estas habilidades, reducen o evitan problemas conductuales y mentales, al tiempo que promueven los resultados académicos positivos a corto y a largo plazo. Los resultados de algunos metaanálisis (Clouder, 2008) refuerzan la idea de que el fomento sistemático de habilidades afectivas y sociales en el ámbito escolar tiene una incidencia positiva, cada vez más reconocida a nivel mundial, pues se previenen problemas de desarrollo, se favorece el despliegue saludable y se mejora el rendimiento académico.

Los hallazgos mencionados apoyan la idea de los beneficios individuales y colectivos de este tipo de destrezas que deben ejercitarse en todo tipo de alumnos, no sólo en los que muestran un comportamiento deficitario, $y$, si es posible, desde la temprana infancia. El progresivo reconocimiento y afianzamiento de psicólogos y pedagogos en los centros educativos puede ser de gran ayuda para animar y mostrar a los educadores cómo desarrollar la inteligencia afectiva en el aula. A continuación se proponen sumariamente algunas vías para enriquecer la estructura cognitivoemocional en la escuela:

- El ejemplo de los educadores.- Los alumnos advierten lo que hacen y dicen los profesores, a quienes tienden a imitar. Aun sin querer, el comportamiento de los educadores se presenta ante los escolares como referencia o base de su conducta. De la misma forma que el ejemplo positivo y rico cala en el educando y le orienta constructivamente ante sí mismo y los demás, el ejemplo negativo penetra en su ser y le arrastra hacia el error. La ejemplaridad de las acciones tiene gran impacto en el niño, especialmente en la forma de organizar la realidad y en el acercamiento a los otros y a cuanto le rodea. La seguridad o temor, el optimismo o pesimismo, las actitudes y valores, el modo de relacionarse, el tono vital, etc., dependen en buena parte de lo que durante la infancia se haya observado e imitado. Un primer axioma ha de ser, por tanto, predicar con el ejemplo, pues es fácil que las conductas sean seguidas. Los niños aprenden a expresar su afectividad observando cómo lo hacen los adultos más cercanos y significativos. La competencia cognitivo-afectiva del profesor influye en el crecimiento intelectual y emocional de sus alumnos.

- La convivencia cordial.- El ambiente que se vive en el aula ha de constituir un medio óptimo para el desarrollo de la inteligencia afectiva. El clima social más apropiado es el que se fundamenta en la cordialidad (del latín, cor, cordis = corazón), es decir, en la comprensión, el respeto, la confianza, la comunicación, la sinceridad y la cooperación. Junto al discurso verbal y las actividades programadas, es decir, lo que podemos llamar educación patente hay otra realidad educativa latente igualmente importante, en la cabe incluir el gesto, la palabra de aliento, etc. Ambas modalidades formativas (explícita e implícita), por más que se presenten con desigual "visibilidad", constituyen 
la atmósfera que troquela al niño. La riqueza emocional del clima escolar opera como poderoso adherente que condiciona la dirección que adopte la conducta infantil.

- Utilizar material que favorezca el desarrollo de la inteligencia afectiva.- Conviene aprovechar situaciones de la vida cotidiana, informaciones de actualidad, películas, etc. Las materias que integran los planes de estudio son adecuadas para trabajar la inteligencia emocional sin desligarla de los demás objetivos educativos. Coincidimos con Bisquerra (2000), cuando afirma que la "educación emocional" debe impregnar todas las áreas académicas a lo largo de toda la escolaridad. En este punto, hemos de avanzar la trascendencia de la lectura. Aun cuando la sociedad de la imagen en que nos encontramos haya desplazado en buena parte al libro, la educación genuina no puede renunciar a la lectura. Al leer se movilizan procesos mentales más complejos que los que habitualmente se activan al recibir mensajes audiovisuales. La experiencia lectora proporciona una oportunidad de acrecentamiento afectivo y personal de la que no se puede prescindir, so pena de rebajar considerablemente la formación.

- Potenciar el razonamiento.- La confrontación de opiniones en un ambiente de reflexión y libertad contribuye a la mejora de la inteligencia afectiva. Hay que evitar, eso sí, caer en el relativismo y en el adoctrinamiento. La infusión de racionalidad desde la infancia ayuda a "poner los pies en la tierra", sin que ello tenga que suponer, en modo alguno, un cercenamiento de la creatividad, tan necesaria como poco valorada aún. La ejercitación en procesos lógico-racionales probablemente ayude a reducir las creencias infundadas de naturaleza supersticiosa o esotérica, que a veces condicionan poderosamente, incluso en los adultos, las relaciones interpersonales. Hay personas cuyas acciones están regidas por interpretaciones carentes de todo fundamento que se nutren plenamente de la subjetividad y a menudo se ligan al "aquí y ahora", de ahí que las generalizaciones sean inapropiadas, cuando no claramente peligrosas. Es preciso favorecer en los alumnos la formación de conceptos sólidos, el enjuiciamiento crítico de la realidad, la curiosidad intelectual y el amor a la verdad.

\subsection{El despliegue de la inteligencia afectiva en la escuela a través de la lectura}

Por su centralidad en este artículo dedicamos un apartado concreto al fomento de la inteligencia afectiva a través de la lectura en la escuela. De hecho, al revisar la intervención psicoeducativa que en materia de inteligencia afectiva se realiza en España, se comprueba que muchos programas encaminados a favorecer el aprendizaje social y emocional en las escuelas se sirven de la lectura como recurso privilegiado. A continuación se citan algunas de las modalidades de lectura más utilizadas:

.Cuentos dedicados a los más pequeños, como el denominado Educación emocional, dirigido a niños de entre 3 y 6 años, de López Cassá (2003) o el Programa de Inteligencia Emocional: Sentir y Pensar de Ibarrola y Delfo (2003), también destinado a los alumnos de educación infantil. 
. Fábulas, narraciones, historias y recortes de prensa, en aquellos programas dirigidos a la Educación Primaria y Secundaria, como Educación Emocional. Programa para la educación primaria (Renom, 2003).

- Libros como el programa de Educación Emocional para la Prevención de la Violencia, de Caruana (2005), dirigido a alumnos de $2^{\circ}$ ciclo de Secundaria, en el que se nos recomienda la lectura del libro ¿Quién se ha llevado mi queso? (Johnson, 1999).

Llegado este punto, podemos preguntarnos: ¿por qué la lectura es un instrumento que posibilita el desarrollo de la inteligencia afectiva?

El poderoso valor de la lectura para mejorar la competencia afectiva de las personas reside en que las historias reflejan muchas de nuestras inquietudes y de nuestras maneras de entender la vida, y esta sensación de realidad es la que nos permite soñar, imaginar, reflexionar, adentrarnos en mundos nuevos y conocer nuevas formas de pensar, sentir y actuar (Morote, 2005).

En efecto, la lectura constituye una senda privilegiada para la dilatación personal. En general, salvo que se trate de textos mal escogidos o de escasa calidad, sus beneficios se dejan sentir en todas las vertientes: cognitiva, afectiva, social, ética, estética y espiritual. La literatura, por tanto, proporciona una experiencia embriagadora y holística, muy apropiada para el cultivo de la inteligencia afectiva. Por desgracia, sin embargo, esta actividad genuinamente cultural, condición indispensable de la educación, se encuentra hoy en claro retroceso (Martínez-Otero, 2010).

Una persona que tiene una buena formación literaria y que ha desarrollado un hábito lector es más libre, más madura y responsable (Tejerina y Rodríguez Gutiérrez, 2005). Mendoza (2005), también incide en este aspecto al afirmar que la lectura es el medio por el que se adquieren muchos de los conocimientos de nuestra formación integral. Por su parte, Janer (2005) indica que la literatura contribuye al proceso de formación de la experiencia humana. La lectura es capaz de liberar al hombre de algunas servidumbres y de algunas dependencias; porque le obliga a una nueva percepción de la vida, al tiempo que abre caminos a la experiencia futura.

Con arreglo a la idea rectora de este artículo, cabe recordar que la lectura es una herramienta formativa que permite alcanzar aprendizajes de índole emocional, moral y social. Marchesi (2005) afirma que la lectura puede transmitir valores socioculturales al mismo tiempo que nos pone en contacto con otros sentimientos, otras experiencias y otras vidas, lo que nos permite superar el individualismo y conectar con las intenciones, las expectativas, los anhelos y las frustraciones de los otros. A su vez, Petit (1999) entiende la lectura como un fenómeno que permite a los individuos aprehender valores articulados y congruentes con el entorno cultural en que se desarrolla.

También la lectura nos permite acrecentar las habilidades intrapersonales, como el autoconocimiento o la motivación. Briz (2002) indica que el alumno que posee hábito 
lector aprende a escuchar, a matizar los sentimientos y las emociones, mejora su autoconcepto, adquiere mayor seguridad en sí mismo y hasta puede superar la timidez. Asimismo, Reyzabal y Tenorio (1994) afirman que la lectura puede modificar y modelar la personalidad.

Pero tenemos que tener en cuenta que no siempre es posible leer para aprender. Para aprender, es necesario comprender, y no basta con una comprensión literal (referida a la recuperación de información explícitamente descrita en el texto), sino que es necesaria una compleja actividad cognitiva a través de la que se procesa la información, una comprensión inferencial (extrapolar información no expresada explícitamente en el texto) (Sacristán, 2008). La simple acción de leer no siempre conlleva la adquisición de conocimientos (leer para aprender). En ocasiones, la falta de comprensión se refleja en las limitaciones que los lectores tienen para generalizar o transferir lo que han aprendido a situaciones diferentes a las que originan su aprendizaje.

Necesitamos "aprender a leer" para poder "leer para aprender". Si leemos para adquirir conocimientos, después de la lectura sabemos algo que no sabíamos, tenemos algo que no teníamos, pero nosotros somos los mismos que antes, nada nos ha modificado (Moreno, 2005). Claro que nosotros podríamos matizar que, aunque somos los mismos, merced al aprendizaje producido, "no somos lo mismo". El aprovechamiento de la experiencia lectora permite el enriquecimiento cognitivoemocional y, por tanto, nos hace mejores. Mas para ello es necesario, como así recogen Benito Morales (2000), Martínez-Otero (1997), Sánchez Miguel (1993) y Beach y Appleman (1984), enseñar a los alumnos estrategias para mejorar la comprensión lectora prestar atención a cómo las emplean para organizar y encauzar su propio aprendizaje (Sacristán, 2008).

En un estudio realizado con población universitaria (García-Lago, 2008), hemos encontrado evidencias de la positiva incidencia de la lectura en la competencia afectiva de las personas. Así, los alumnos que leen de manera habitual presentan mejores habilidades socioemocionales que aquellos que lo hacen de un modo ocasional. Estos datos refuerzan los encontrados por Martínez Serrano y Sierra (2005), quienes demostraron que el material de autoayuda tenía beneficioso impacto en el tratamiento de distintos problemas como el trastorno de pánico (Carlbring, Westling y Andersson, 2000), la depresión, (Floyd, 2003; McKendree-Smith, Floyd y Scogin, 2003), el tabaquismo (García y Becoña, 1994; Míguez Varela, 2004) y el alcoholismo (Barber y Gilbertson, 1998). Estos positivos efectos, que acaso pueden matizarse a partir de diversas variables, no se hallaron en otro tipo de problemas.

Como cabe suponer, no es empresa fácil ofrecer recomendaciones válidas para todas las situaciones. Los profesores deben encontrar sus propias vías para cultivar la inteligencia afectiva. En realidad, siempre se ha hecho, aunque a menudo de modo inconsciente y con resultados imprevistos cuando no claramente adversos. Es preferible, pues, adoptar una perspectiva científica, lo que es tanto como establecer objetivos y acción sistemática. Se sabe que la inteligencia afectiva favorece el aprendizaje, la maduración y el bienestar personal, por lo que hay que desear que en 
los centros escolares se tome conciencia de la necesidad de promover esta capacidad de los educandos como senda que permita avanzar hacia la autorrealización y la convivencia. Sin duda alguna, una vía regia es la lectura.

\section{CONCLUSIONES}

La escuela debe comprometerse más en el desarrollo de la competencia emocional de los niños, puesto que, los resultados de las distintas investigaciones revelan que el cociente intelectual (CI), en otro tiempo sobrevalorado, desempeña un papel más modesto que la inteligencia afectiva a la hora de explicar el éxito en la vida. El CI se erige, por tanto, como condición necesaria pero no suficiente, mientras que el "cociente emocional" se revela como fundamental y mejor predictor del éxito personal.

La lectura es una herramienta al alcance de maestros/profesores que puede enriquecer tanto los procesos cognitivos como los afectivos. La lectura es una actividad instrumental básica de vital importancia ya que a través de ella la persona no sólo accede al léxico y comprende el texto, sino que aprehende lo que en él está escrito y adquiere o afianza valores, conocimientos y destrezas. Así, por ejemplo, el pequeño lector puede adentrarse en otras vidas y captar, siquiera sea de modo inconsciente, el acontecer existencial de personajes, algunos de los cuales le parecerán maravillosos, y obtener un gran beneficio formativo, advertido en los conocimientos adquiridos, en las emociones despertadas, en los valores transmitidos y, por supuesto, en el saludable disfrute.

La lectura como herramienta que permite incrementar la competencia emocional en el alumnado se nos muestra como un ámbito que hay que seguir explorando. Aunque hay cada vez más voces que reclaman cultivar a un tiempo la cabeza y el corazón, a lo largo de este trabajo se evidencia la necesidad de continuar investigando en este rico campo de la inteligencia afectiva, así como en sus implicaciones formativas.

En este sentido, aceptando la idea defendida en este artículo de que la lectura juega un relevante papel en el desarrollo cognitivo-emocional del educando, sería conveniente contar con más y mejores selecciones de obras literarias. Estamos convencidos de que una labor así, todo lo abierta y flexible que se quiera, constituye un jalón fundamental en el enriquecimiento de la inteligencia afectiva y, por ende, en el desarrollo personal y social.

\section{BIBLIOGRAFÍA}

Barber, J. B. y Gilbertson, R. (1998). Evaluation of a selfhelp manual for the female partners of heavy drinkers. Research on Social Work Practice, 8, 141-151.

Beach, R., y D. Appleman. (1984). Reading strategies for expository and literary text types. En A. C. Purves y O. S. Niles (eds.), Becoming readers in a complex society. Eighty-third yearbook of the National Society for the Study of Education. (p. 115-143). Chicago: University of Chicago Press. 
Benito Morales, F. (2000). La alfabetización en información en centros de primaria y secundaria. En J. Gómez (Coord.), Estrategias y modelos para enseñar a usar la información. (p. 79-130). Murcia: KR.

Bisquerra, R. (2000). Educación emocional y bienestar. Barcelona: Praxis.

Briz, E. (2002). Evaluación formativa de la lectoescritura: las destrezas de la lectura expresiva. En MaS. Santos y Map. Soler (Coords.), Investigaciones sobre el inicio de la lectoescritura en edades tempranas. (p. 177-199). Madrid: MECD.

Caruana, A. (Coord.). (2005). Programa de educación emocional para la prevención de la violencia. Valencia: Generalitat Valenciana. Consejería de Cultura, Educación, y Deporte.

Castilla del Pino, C. (2000). Teoría de los sentimientos. Barcelona: Tusquets.

Clouder, C. (Coord.).(2008). Educación emocional y social. Análisis internacional. Extraído el 11 octubre, 2008 del sitio Web http://educacion.fundacionmbotin.org/ficheros_descarga/pdf/ES/dossier.pdf

Floyd, M. (2003). Bibliotherapy as an adjunt to psychotherapy for depression in older adults. Journal of Clinical Psychology, 59, 187.

García, M. P y Becoña, E. (1994). Manuales de autoayuda para dejar de fumar: una revisión. Adicciones: Revista Socidrogalcohol, 6, 73-89.

García-Lago, V. (2008). Influencia de la lectura en el desarrollo de las competencias emocionales: un estudio hecho con población universitaria.

Trabajo de investigación inédito. Madrid: Universidad Camilo José Cela.

Goleman, D. (1997). Inteligencia emocional. Barcelona: Kairós.

Goleman, D. (2000). La práctica de la inteligencia emocional. Barcelona: Kairós.

Hersh, R; Reimer, J. y Paolitto, D. (1988). El crecimiento moral. De Piaget a Kohlberg. Madrid: Narcea.

Ibarrola, B. Y Delfo, E (2003). Sentir y pensar. Programa de Inteligencia emocional para niños de 3 a 5 años. Madrid: SM.

Janer, G. (2005). No hay espectáculo más hermoso. En Revista de Educación, número extra 2005. 179-188.

Johnson, S. (1999). ¿Quién se ha llevado mi queso? Barcelona: Urano.

Kohlberg, L. (1992). Psicología del desarrollo moral. Bilbao: Desclée de Brouwer. 
López Cassá, E. (2003). Educación emocional. Programa para 3-6 años. Barcelona: Praxis.

Marchesi, A. (2005). La lectura como estrategia para el cambio educativo. En Revista de Educación, número extra 2005. 15-36.

Martínez Serrano, P. y Sierra, J.C. (2005). Efecto de la lectura del material de autoayuda sobre algunas variables psicológicas en una muestra no clínica. Extraído el 29 Septiembre 2008 del sitio Web

http://www.scielo.org.co/scielo.php?script=sci_arttext\&pid=S16579267200500020000 7\&lng=es\&nrm=iso\&tlng=es

Martínez-Otero, V. (1997). Los adolescentes ante el estudio. Causas y consecuencias del rendimiento académico. Madrid: Fundamentos.

Martínez-Otero, V. (2007). La inteligencia afectiva. Teoría, práctica y programa. Madrid: CCS.

Martínez-Otero, V. (2010). Literatura y educación. Madrid: CCS.

McKendree-Smith, N. L., Floyd, M. \& Scogin, F. R. (2003). Self-administered treatment for depression: A review. Journal of Clinical Psychology, 59, 275.

Mendoza, A. (2005). Notas introductorias sobre la lectura y la formación lectora. En L.J. Tigero (Coord.), La seducción de la lectura en edades tempranas. (p. 21-37) Madrid: MECD.

Míguez Varela, M. C. (2004). Los procedimientos de autoayuda para dejar de fumar. Adicciones: Revista de Socidrogalcohol, 16 (supl. 2), 339-357.

Moreno, V. (2005). Lectores competentes. En Revista de Educación, número extra 2005. 153-169.

Morote, P. (2005). El cuento de tradición oral y el cuento literario: de la narración a la lectura. En L.J. Tigero (Coord.), La seducción de la lectura en edades tempranas. (p. 159-199) Madrid: MECD.

Petit, M. (1999). Nuevos acercamientos a los jóvenes y a la lectura. México: Fondo de Cultura Económica.

Piaget, J. (1973). Seis estudios de psicología. Barcelona: Barral.

Piaget, J. (2001). Inteligencia y afectividad. Buenos Aires: Aique.

Reyzabal, Ma.V. y Tenorio, P. (1994). El aprendizaje significativo de la literatura. Madrid: La Muralla.

Rojas, E. (1988). El laberinto de la afectividad. Madrid: Espasa Calpe. 
Sacristán, F. (2008). Diferencias cognitivas en el proceso de aprendizaje de la lectura. Revista Española de Orientación y Psicopedagogía, 19 no1, 13-22.

Sánchez Miguel, E. (1993) Los textos expositivos: estrategias para mejorar su comprensión. Madrid: Santillana

Tejerina, I. y Rodríguez Gutiérrez, B. (2005). Lecturas y lectores en la ESO: una investigación educativa. Santander: Ediciones de la Consejería de Educación. Gobierno de Cantabria.

Renom, A. (2003). Educación emocional. Programa para la educación primaria. Barcelona: Praxis.

Zubiri, X. (1991). Inteligencia sentiente. Madrid: Alianza Editorial-Fundación Xavier Zubiri.

\section{Valentín Martínez-Otero Pérez}

Doctor en Psicología (UCM) y Doctor en Pedagogía (UNED), con Premio Extraordinario. Profesor Titular del Departamento de Teoría e Historia de la Educación (Facultad de Educación, UCM). Autor de numerosos libros y publicaciones sobre temas educativos.

\section{Virginia García-Lago Ibáñez}

Licenciada en Psicopedagogía con el Primer Premio Nacional de Fin de Carrera del MEC y Premio Extraordinario de la Universidad Complutense de Madrid. Actualmente trabaja como maestra en el Colegio Santa María (Collado Villalba, Madrid). 